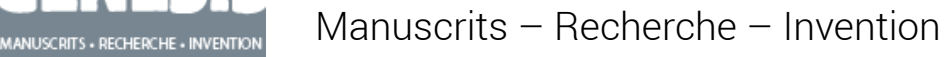

$53 \mid 2021$

Sartre Beauvoir. Genèses croisées

\title{
Baudelaire, éditeur des « Fleurs du mal »
}

\section{Andrea Schellino}

\section{OpenEdition}

\section{Journals}

Édition électronique

URL : https://journals.openedition.org/genesis/6277

DOI : 10.4000/genesis.6277

ISSN : 2268-1590

\section{Éditeur :}

Presses universitaires de Paris Sorbonne (PUPS), Société internationale de génétique artistique littéraire et scientifique (SIGALES)

\section{Édition imprimée}

Date de publication : 15 décembre 2021

Pagination : 195-199

ISBN : 979-10-231-0716-6

ISSN : 1167-5101

\section{Référence électronique}

Andrea Schellino, «Baudelaire, éditeur des «Fleurs du mal » », Genesis [En ligne], 53 | 2021, mis en

ligne le 15 décembre 2021, consulté le 12 janvier 2023. URL : http://journals.openedition.org/genesis/ 6277 ; DOI : https://doi.org/10.4000/genesis.6277 


\title{
Baudelaire, éditeur des «Fleurs du mal»
}

\author{
Andrea Schellino
}

$\mathrm{U}$ ne longue tradition éditoriale relie les premières publications des œuvres de Baudelaire aux rééditions les plus récentes. C'est une histoire marquée par une étonnante continuité. En 1887, Eugène Crépet, qui avait impliqué Baudelaire dans le projet des Poètes français (1861-1862), publie les Euvres posthumes et correspondances inédites chez Quantin. En reprenant le flambeau, son fils Jacques sera le maître d'œuvre d'une monumentale édition des Euvres complètes de Baudelaire, parue en dix-neuf volumes chez Louis Conard, entre 1922 et 1953, édition à laquelle a contribué Claude Pichois.

La nouvelle édition des Euvres complètes de Baudelaire dans la Bibliothèque de la Pléiade, dont la parution est prévue en 2023, s'inscrit à la fois dans cette tradition et dans l'histoire des éditions Gallimard : c'est un volume d'œuvres poétiques de Baudelaire qui inaugure la collection, en 1931, suivi en 1932 d'un volume d'œuvres critiques, établi et annoté par Yves-Gérard Le Dantec. En 1961, Claude Pichois a révisé, complété et refondu en un seul volume d'Euvres complètes ces deux tomes, avant de publier, en 1973, une édition de la Correspondance de Baudelaire, et une nouvelle édition des Euvres complètes, en 1975-1976.

Les éditeurs de Baudelaire, et particulièrement les éditeurs des Fleurs $d u$ Mal, se confrontent dans leur travail à un remarquable modèle. Sa publication est étroitement liée à l'exigence créative de Baudelaire. L'éditeur a doublé, en quelque sorte, le poète. Avant même d'en confier le soin à Auguste Poulet-Malassis, Baudelaire a été l'éditeur scrupuleux de sa propre poésie. La longue attente qui a précédé la publication des Fleurs du Mal, participant à la naissance d'une aura mythique autour du jeune poète, n'a fait que renforcer l'idée que ce livre, «fait avec fureur et patience1 ${ }^{1}$, était destiné à rester.

Tout lecteur des Fleurs du Mal connaît l'acribie et la patience dont le poète a fait preuve dans la correction de ses textes, dans leur préparation, dans leur mise en page et dans la vérification des détails typographiques. Baudelaire a consacré à la publication de ses premiers poèmes et des deux éditions des Fleurs du Mal la même attention qu'il avait réservée à leur écriture. Lorsqu'il corrige les placards de son recueil, en avril 1857, il s'adresse à Poulet-Malassis pour lui rappeler «qu'en toute espèce de production», il n'y a «d'admissible que la perfection $»^{2}$. Le souci de perfection hante Baudelaire. «Sitôt que vous voulez me donner l'idée d'un parfait artiste, mon esprit ne s'arrête pas à la perfection dans un genre de sujets, mais il conçoit immédiatement la nécessité de la perfection dans tous les genres ${ }^{3}$ », écrit-il dans sa notice sur Victor Hugo. La perfection déborde la forme poétique, pour tendre à la lisibilité qu'assure une publication irréprochable.

Malgré les témoignages de ses amis de jeunesse, comme Ernest Prarond, un mystère enveloppe la genèse des premiers poèmes composés par Baudelaire, recueillis en 1857 dans Les Fleurs du Mal. Le premier poème qu'il publie, «À une Créole», qui deviendra plus tard «À une dame créole», trouve place dans L'Artiste, le 25 mai 1845; le 13 décembre 1846, dans la même veine, il fait paraître «À une Malabaraise». Baudelaire fait aussi annoncer la future parution des Lesbiennes, titre qui apparait au second plat de la couverture de L'Agiotage de Pierre Dupont, en octobre 1845 , et qui réapparaît à d'autres reprises jusqu'au début de 1847. Qu'aurait-il dû contenir ce recueil au titre sulfureux, évoquant le thème saphique et l'amour interdit? En février 1849, Baudelaire lui préfère un autre titre, Les Limbes, recueil de «poésies» annoncé dans L'Écho des marchands de vins, et «destiné à représenter l'histoire des agitations et les mélancolies de la jeunesse moderne». Le livre aurait dû paraître le 24 février 1849 chez Michel Lévy, le futur éditeur des œuvres de Baudelaire, entre 1868 et 1870 . Le recueil semble alors glisser vers l'incertitude

1. Lettre de Baudelaire à Mme Aupick, 9 juillet 1857; dans Correspondance, éd. cit., t. I, p. 411.

2. Lettre de Baudelaire à Auguste Poulet-Malassis; ibid., t. I, p. 393.

3. Charles Baudelaire, Euvres complètes, désormais $E C$, texte établi, présenté et annoté par Claude Pichois, Paris, Gallimard, coll. «Bibliothèque de la Pléiade», 1976, t. II, p. 135. 
ou l'attente théologique, qui ont été associées, par certains interprètes de l'œuvre de Baudelaire, aux périodes limbiques identifiées par Charles Fourier, genèse de la société et de l'industrie. La marginalité des limbes traduit également la marginalité d'un projet éditorial qui ne se réalise pas encore.

C'est pendant cette période que Baudelaire aurait fait exécuter une copie calligraphiée de ses vers par M. Palis, où s'annonce déjà le goût pour la perfection qu'il manifestera lors de la publication de son recueil. Cet exemplaire avait suscité en 1850 l'admiration d'un de ses amis, Charles Asselineau, qui écrit dans sa biographie :

C'est alors qu'il me montre chez lui [...] le manuscrit de ses poésies magnifiquement copié par un calligraphe, et qui formait deux volumes in-4o cartonnés et dorés. C'est ce manuscrit qui a servi pour l'impression des Fleurs du Mal${ }^{4}$.

Ce manuscrit «magnifiquement copié» ne trouva pas grâce aux yeux de Baudelaire, qui s'en était plaint auprès de Narcisse Ancelle, le 10 janvier 1850 :

Des fautes ridicules et folles, commises dans la Table, comme Le Tombant vivant, Vitesse de la lune, pour Le Tombeau vivant, Tristesse de la lune, et bien d'autres; la dorure pleine de taches, la reliure qui devait être en chagrin et qui est en papier imitant le chagrin, des corrections indiquées par moi au crayon et qui n'ont pas été accomplies, témoignent qu'il a profité de mon absence pour ne pas faire son devoir, de plus, pour me voler 5 .

La «nuée de fautes» du manuscrit lui parait inconciliable avec la dignité de ses vers et l'attention qu'il avait portée à leur rédaction.

Plusieurs poèmes de Baudelaire paraissent dans la presse ou dans des romans entre 1852 et 1855 . Mais le poète, dont la réputation grandit, caresse l'idée d'une publication plus ambitieuse, rassemblant un nombre consistant de poèmes dans l'une des revues littéraires les plus lues de l'époque : la Revue des deux mondes. Son directeur, François Buloz, a déjà fermé plusieurs fois à Baudelaire les portes de la revue. Cinq ans plus tard, le poète se proposera d'écrire un «beau tableau» sur «la canaille littéraire», constituée en particulier des journalistes et des patrons de presse. Mais en juin 1855 Baudelaire parvient à convaincre Buloz de faire paraître dans la Revue des deux mondes dix-huit poèmes, sous le titre Les Fleurs $d u$ Mal : «Au lecteur», «Réversibilité», «Le Tonneau de la Haine», «La Confession», «L'Aube spirituelle», «La Volupté», «Voyage à Cythère», «À la belle aux cheveux d'or», «L'Invitation au voyage», «Moesta et errabunda», «La Cloche», «L'Ennemi», «La Vie antérieure», «Le Spleen», «Remords posthume», «Le Guignon», «La Béatrice», «L'Amour et le Crâne». Cette publication préoriginale, accompagnée d'une note par Émile Montégut qui réduit les vers de Baudelaire à l'expression des « défaillances » et des «douleurs morales » du temps, a une importance capitale : deux ans plus tard paraitra la version en volume de ces mêmes Fleurs du Mal, chez Poulet-Malassis et De Broise (fig. 1 et 2). Les dix-huit poèmes laissent place aux cent poèmes de l'édition en volume, enrichis d'une pièce liminaire adressée «Au lecteur». À l'architecture étudiée du micro-recueil de la Revue des deux mondes succède $l^{\prime}$ «architecture secrète ${ }^{6}$ » du volume.

Poulet-Malassis est un éditeur éclairé, héritier des plus belles traditions : hostile aux éditions parisiennes à grands tirages, il affectionne les procédés anciens, imprime sur papier vélin ou vergé, recherche l'élégance typographique par l'association d'encre noire et d'encre rouge, que Baudelaire choisira cependant de limiter à la page de titre. Éditeur confidentiel, ne pouvant rivaliser avec ses grands concurrents, comme Michel Lévy ou Hachette, il est prêt à revêtir la poésie des Fleurs $d u$ Mal de la forme typographique que souhaite Baudelaire.

Le contrat entre le poète et l'éditeur alençonnais est signé le 30 décembre 1856. Le manuscrit est remis le 6 février 1857, comme Baudelaire l'annonce dans la foulée à sa mère. C'est à ce moment qu' on peut le mieux comprendre la ferveur qu'il met à cette publication. Face à son éditeur, qui restera patient devant ses incessantes requêtes, l'auteur des Fleurs du Mal ne cessera de vouloir se substituer à lui, pour exercer un contrôle minutieux, exigeant et radical sur toutes les étapes d'édition de son recueil. Le ton est donné dès le 10 février 1857, lorsque Baudelaire

4. Charles Asselineau, Charles Baudelaire, sa vie et son æuvre, Paris, Alphonse Lemerre, 1869, p. 37.

5. Charles Baudelaire, Correspondance, éd. cit., t. I, p. 158.

6. Jules Barbey d'Aurevilly, «Les Fleurs du Mal, par M. Charles Baudelaire», dans Articles justificatifs pour Charles Baudelaire auteur des Fleurs du Mal, [août 1857]; rééd. dans André Guyaux, Un demi-siècle de lectures des Fleurs du Mal (1855-1905), Paris, Presses de 1'Université Paris-Sorbonne, coll. «Mémoire de la critique», 2007, p. 191-198. 


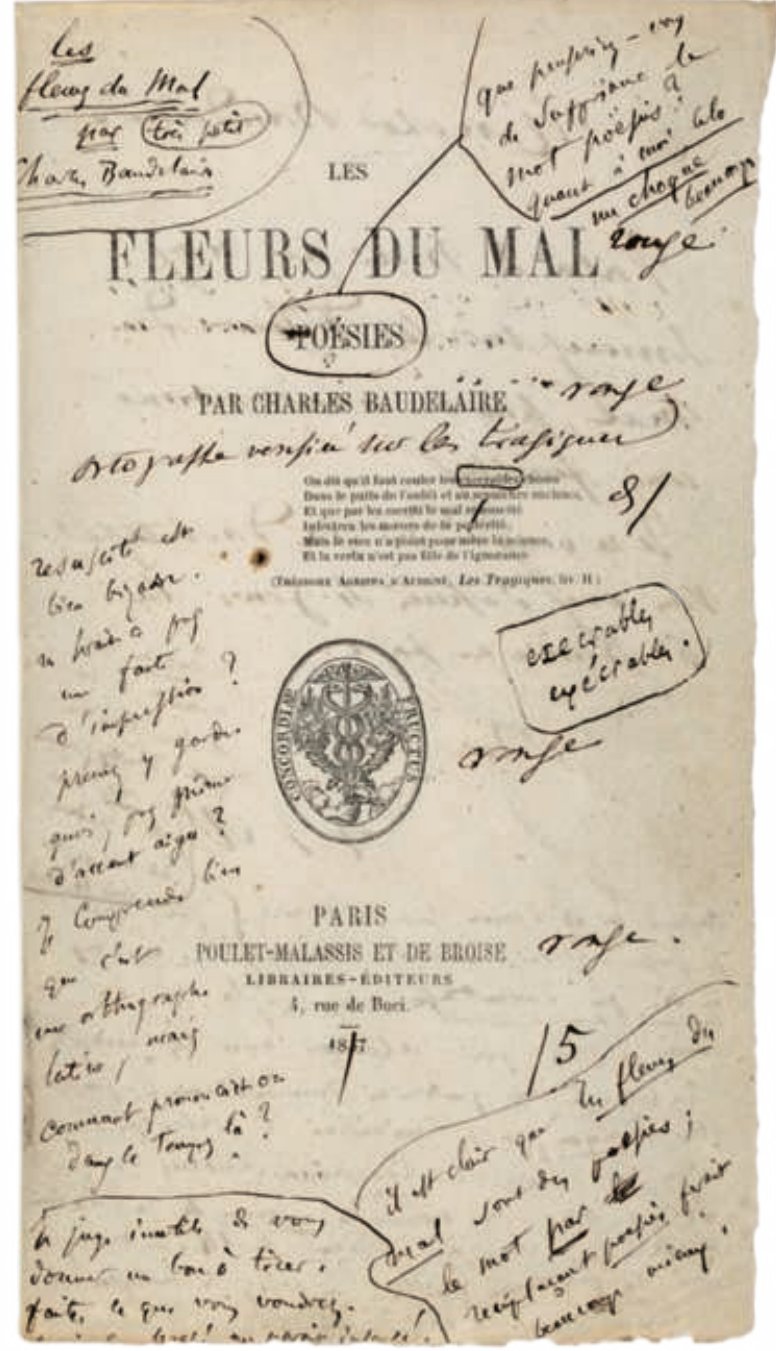

Fig. 1 : Les Fleurs du Mal, épreuves corrigées de l'édition de 1857, page de titre.

(C) BnF, Réserve des livres rares, RES P-YE-3006

demande à Poulet-Malassis «une épreuve-placard et une épreuve mise en pages ${ }^{7}$ », et ces mêmes épreuves toujours en double exemplaire. La «question typographique», que Baudelaire prétend ne pas maîtriser, apparaît au contraire au cœur de ses préoccupations :

Quant à la question typographique, je n'y entends rien, ou du moins je n'y entends qu'avec mon œil.

Je vous recommande seulement, lors de la mise en page, de ne pas être avare de blancs, et puis de composer la Dédicace dans un certain style solennel que vous saurez trouver, grâce à votre excellent goût. Cependant, il serait peut-être bon de ne pas donner à un manuscrit moderne les archaïsmes et les gentillesses du rouge. Pas de coquetterie ${ }^{8}$.

Le 7 mars, Baudelaire reviendra sur la question de l'orthographe, indiquant sa prédilection pour «l'ancienne,

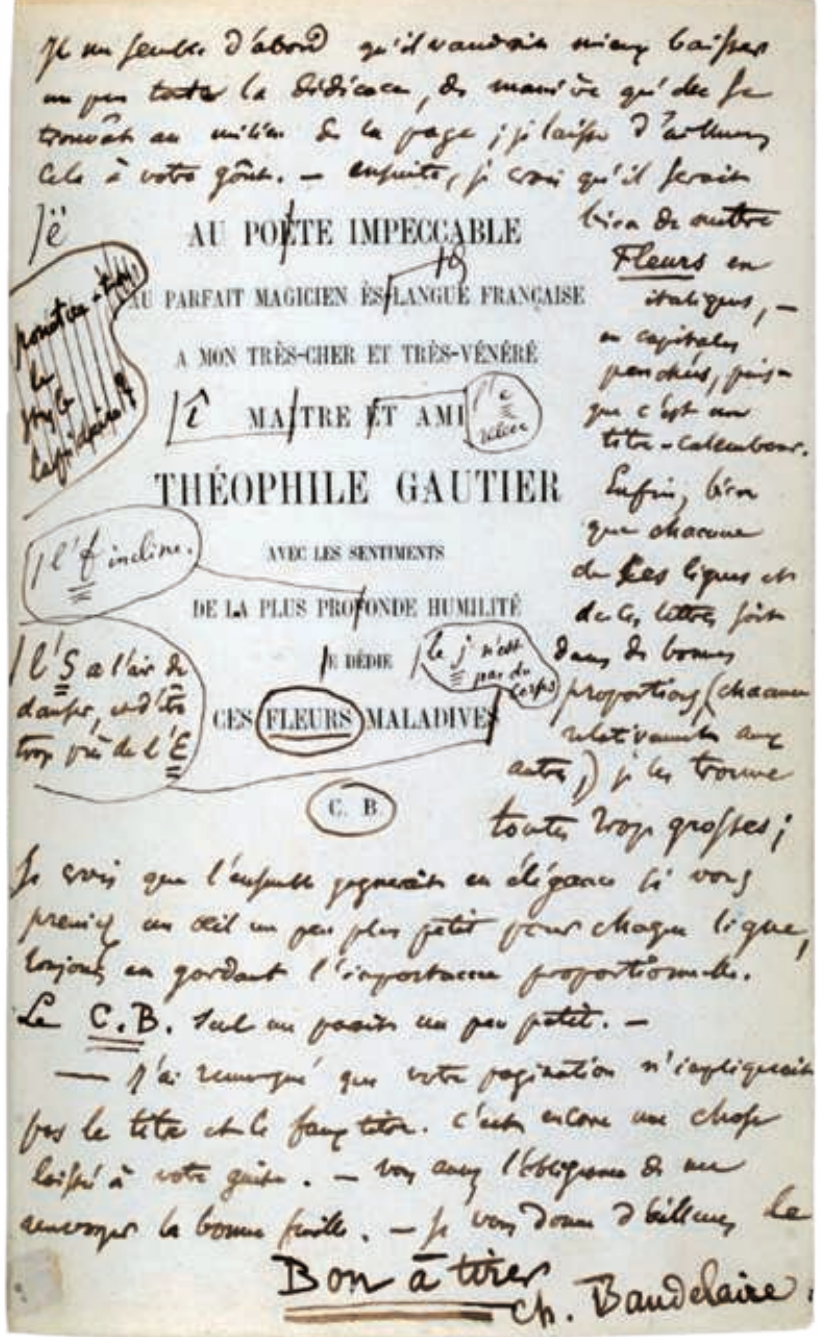

Fig. 2 : Les Fleurs du Mal, épreuves corrigées de l'édition de 1857, dédicace.

CC BnF, Réserve des livres rares, RES P-YE-3006

mais modérée ${ }^{9} »$. Il se conforme ainsi à l'usage des traits d'union dans les superlatifs construits avec très et un adjectif, selon une pratique répandue au milieu du siècle, tandis qu'il écrit «poème» et «poète» avec l'accent grave, à la différence de ce qu'il fera en 1861, quand il préférera revenir à la graphie traditionnelle, avec tréma.

La correction des placards et des épreuves occupe Baudelaire et son éditeur pendant plusieurs mois. Les envois se multiplient, entre février et juin 1857. Baudelaire se trouve à Paris pour suivre la publication des Aventures d'Arthur Gordon Pym dans Le Moniteur universel. Puisqu'il

7. Charles Baudelaire, Correspondance, éd. cit., t. I, p. 374.

8. Ibid.

9. Ibid., t. I, p. 378 . 
souhaite parfois supprimer ou intercaler de nouveaux poèmes dans son recueil, il faut régulièrement recommencer le travail des épreuves. Poulet-Malassis suit son auteur jusqu'au bout, porté par l'admiration qu'il éprouve pour l'ouvrage à paraître. Baudelaire nourrit des craintes quant à l'épaisseur du recueil de cent un poèmes, qui ne doit absolument pas, dans son esprit, paraître une «plaquette». Pour pallier cet inconvénient, il avait insisté auprès de son imprimeur afin que celui-ci publiât ses poèmes en corps 9 et non en corps 8 , qui lui avait paru trop petit pour un recueil de poésie. Les 250 pages finales des Fleurs du Mal, dans un volume en format in-12, le rassurent.

Nous pouvons suivre une partie du chemin qui conduit du manuscrit confié à Poulet-Malassis au volume imprimé des Fleurs du Mal, grâce aux lettres que Baudelaire envoie à son éditeur, et surtout grâce au recueil d'épreuves acquis par la Bibliothèque nationale de France en 1998. Le dossier n'est malheureusement pas complet, mais quelques passages importants s'y trouvent, comme la correction de la dédicace à Théophile Gautier. Le poète se fait éditeur de lui-même. Comme l'observent Jacques Dupont et Claude Pichois, «il ne s'agit pas seulement d'un travail qui relève du créateur : modification d'un mot ou d'un vers, correction de fautes d'impression [...], questions d'orthographe, lettres cassées ou mal venues, mais aussi de détails techniques qui sont du domaine du correcteur professionnel et auxquels la plupart des écrivains sont insensibles $10_{\gg}$. La question des blancs typographiques et des espaces préoccupe Baudelaire autant que celle de la dimension des caractères, comme s'il s'agissait de pauses musicales. Ainsi, le titre courant du premier vers doit, à ses yeux, se séparer de la suite du poème; les blancs entourant les poèmes sans strophes font, quant à eux, l'objet d'une attention particulière.

Le volume est mis en vente le 25 juin 1857, au prix de trois francs; les grands papiers coûtent six francs. Le tirage est alors fixé à 1100 exemplaires environ. L'auteur et l'éditeur minutieux des Fleurs du Mal n'auront su éviter quelques coquilles, qui varient d'ailleurs d'exemplaire à exemplaire, selon le tirage. Aux pages 31 et 108 du volume, le titre courant indique par exemple Feurs au lieu de Fleurs. Mais l'acribie de Baudelaire le poussera jusqu'à corriger, plume à la main, ces imprécisions dans certains exemplaires du recueil qu'il distribuera à des amis et à des connaissances.
On ne sera pas surpris en constatant que la parution de l'édition originale des Fleurs du Mal, loin d'épuiser l'aventure éditoriale de la poésie de Baudelaire, n'en est que le principe. La condamnation qui mutile en août 1857 le recueil mène le poète à rêver d'une nouvelle forme d'aboutissement pour Les Fleurs du Mal, rêve qui ne se réalisera ni du vivant de Baudelaire, ni après sa mort. La seconde édition des Fleurs du Mal - et il faut bien dire «seconde», selon ce qu' on lit sur la page de titre de l'édition - et puisque Baudelaire n'en publiera pas de troisième - paraîtra encore chez Poulet-Malassis, en février 1861. Le volume contient les cent un poèmes de l'édition de 1857 , moins les six pièces condamnées. Il s'enrichit de trente et un poèmes publiés par Baudelaire depuis 1857, plus «La Fin de la journée», qui est inédit, et d'une nouvelle section, «Tableaux parisiens ». Poulet-Malassis recourt cette fois-ci à un imprimeur parisien, Simon Rançon. Le déroulement de cette publication nous reste inconnu, puisque ni les échanges épistolaires entre Baudelaire et Poulet-Malassis, ni les épreuves de cette seconde édition des Fleurs du Mal n'ont été conservés. Une lettre que Baudelaire envoie le 20 novembre 1860 au correcteur Rigaud laisse entrevoir que le poète-éditeur n'avait pas réduit ses exigences :

Je serai bientôt hors d'état, mon cher Rigaud, de semer des points et des virgules, de retourner des lettres, de rétablir des mots dans les épreuves que vous me retournez. Quand, dans Petites Vieilles, vous me faites dire : sornettes pour Sonnettes, italiens pour citadins, je vous trouve vraiment trop peu zélé pour l'éclosion de nos Fleurs ${ }^{11}$.

L'œil de Baudelaire porte ses fruits : l'édition des Fleurs du Mal de 1861 sera moins fautive que l'édition de 1857. Mais cette publication n'interrompt pas l'élan éditorial qui tient tant à cœur au poète : à l'été 1861, Poulet-Malassis prévoit une édition de luxe du recueil, présentée à l'Exposition universelle de Londres de 1862. Baudelaire rêve alors d'une «édition définitive augmentée de quarante-deux poèmes nouveaux ». On remarquera le mot définitive, que Baudelaire associera dans les dernières années de sa vie au projet d'une troisième édition des Fleurs du Mal, destinée

10. Claude Pichois, Jacques Dupont, L'Atelier de Baudelaire : "Les Fleurs $d u$ Mal», édition diplomatique, Paris, Honoré Champion, 2005, t. I, p. 39. 11. Charles Baudelaire, Correspondance, éd. cit., t. II, p. 105-106. 
non plus à ses contemporains, mais à la postérité. On sait que les dernières pensées du poète, encore conscient, allaient à cette édition tant désirée : «préoccupé, voire obsédé» par son sort, selon le mot de Jacques Dupont et de Claude Pichois ${ }^{12}$, il cherche un éditeur, et prépare un exemplaire pour cette nouvelle version de son chef-d'œuvre, sans toutefois que les nouveaux poèmes soient définitivement distribués dans le recueil. Ce seront ses amis, en particulier Charles Asselineau et Théodore de Banville, qui se chargeront de publier la troisième version des Fleurs $d u$ Mal, qui paraîtra, posthume, dans le premier tome des Cuvres complètes chez Michel Lévy, en décembre 1868.

12. Claude Pichois, Jacques Dupont, L'Atelier de Baudelaire : «Les Fleurs du Mal», éd. cit., p. 62.

ANDREA SCHELlino est docteur de l'Université Paris-Sorbonne, où il a soutenu en 2017 une thèse sur Le Concept de décadence de Baudelaire à Nietzsche (Classiques Garnier, 2020). Coresponsable du groupe Baudelaire de l'ITEM (CNRS-ENS), il est chercheur à l'Université Rome III. Il a collaboré à l'édition des Romans et nouvelles (2019) de Joris-Karl Huysmans et des CEuvres (2020) de Victor Segalen dans la Bibliothèque de la Pléiade. Il codirige, pour la même collection, la nouvelle édition des CEuvres complètes de Baudelaire. 